\title{
Aging Affects Hemispheric Asymmetry in the Neural Representation of Speech Sounds
}

\author{
Teri James Bellis, ${ }^{1,2}$ Trent Nicol, ${ }^{1}$ and Nina Kraus ${ }^{1,3}$ \\ ${ }^{1}$ Auditory Neuroscience Laboratory, Department of Communication Sciences and Disorders, and ${ }^{3}$ Departments of \\ Neurobiology and Physiology and Otolaryngology, Northwestern University, Evanston, Illinois, and 2Department of \\ Communication Disorders, University of South Dakota, Vermillion, South Dakota
}

Hemispheric asymmetries in the processing of elemental speech sounds appear to be critical for normal speech perception. This study investigated the effects of age on hemispheric asymmetry observed in the neurophysiological responses to speech stimuli in three groups of normal hearing, right-handed subjects: children (ages, 8-11 years), young adults (ages, 20-25 years), and older adults (ages $>55$ years). Peak-to-peak response amplitudes of the auditory cortical P1-N1 complex obtained over right and left temporal lobes were examined to determine the degree of left/right asymmetry in the neurophysiological responses elicited by synthetic speech syllables in each of the three subject groups. In addition, mismatch negativity (MMN) responses, which are elicited by acoustic change, were obtained. Whereas children and young adults demonstrated larger P1-N1-evoked response amplitudes over the left temporal lobe than over the right, responses from elderly sub- jects were symmetrical. In contrast, MMN responses, which reflect an echoic memory process, were symmetrical in all subject groups. The differences observed in the neurophysiological responses were accompanied by a finding of significantly poorer ability to discriminate speech syllables involving rapid spectrotemporal changes in the older adult group. This study demonstrates a biological, age-related change in the neural representation of basic speech sounds and suggests one possible underlying mechanism for the speech perception difficulties exhibited by aging adults. Furthermore, results of this study support previous findings suggesting a dissociation between neural mechanisms underlying those processes that reflect the basic representation of sound structure and those that represent auditory echoic memory and stimulus change.

Key words: laterality; cerebral dominance; aging; speech perception; evoked potentials; mismatch negativity
Hemispheric asymmetries have been linked to a variety of perceptual and language functions, including the processing of elemental acoustic features of speech in humans (Phillips and Farmer, 1990; Sharma et al., 1994) and in animal models (Fitch et al., 1993). The finding that individuals with left hemisphere cortical damage demonstrate deficits in the perception of speech signals supports the importance of the left hemisphere for normal speech perception (Auerbach et al., 1982; Phillips and Farmer, 1990). Moreover, abnormal patterns of asymmetry have been linked to language-learning problems in children (Obrzut et al., 1983; Dawson et al., 1989).

Studies of the neurophysiological representation of acoustic stimuli have demonstrated that stimuli with complex speech-like acoustic properties, including rapid spectrotemporal changes, yield greater activation in auditory cortex over the left hemisphere (Elmo, 1987; Zatorre et al., 1992; Belin et al., 1998). This asymmetry in the neurophysiological representation of basic speech signals has been shown to occur even at the thalamic level and regardless of whether binaural or monaural stimulation is used (King et al., 1999).

It has been suggested that age-related changes in the pattern of

\footnotetext{
Received Aug. 23, 1999; revised Oct. 18, 1999; accepted Oct. 27, 1999.

This work was supported by the National Institutes of Health National Institute on Deafness and Other Communicative Disorders Grant DC01510 and the Foundation for Hearing and Speech Research. Special thanks to Dawn Burton Koch for her invaluable support and editorial assistance.

Correspondence should be addressed to Dr. Teri James Bellis, Department of Communication Disorders, University of South Dakota, 414 East Clark Street, Vermillion, SD 57069. E-mail: tbellis@usd.edu.

Copyright (C) 2000 Society for Neuroscience $0270-6474 / 00 / 200791-07 \$ 15.00 / 0$
}

hemispheric asymmetry may underlie some of the auditory perceptual difficulties experienced by aging adults (Jerger and Jordan, 1992; Marvel et al., 1992; Jerger et al., 1994; Pekkonen et al., 1995). The purpose of this study was to investigate whether age affects the degree of left/right hemispheric asymmetry in the neural representation of monaurally presented speech stimuli. A further purpose was to determine whether the ability to make fine-grained acoustic discriminations of speech signals was affected by age.

\section{MATERIALS AND METHODS}

\section{Subjects}

Subjects for this study consisted of very young (ages, 8-11 years), young adult (ages, 20-25 years), and older adult (ages $>55$ years) right-handed females with no history of neurological or otological disease or trauma. All subjects evidenced normal peripheral hearing sensitivity, defined as pure-tone thresholds $<25 \mathrm{~dB}$ for octave frequencies of $500-8000 \mathrm{~Hz}$. All subjects were paid for their participation.

\section{Evoked potential stimulus and recording parameters}

Evoked potential electrophysiological responses reflect processes that require synchronous activity across populations of neurons. Two types of auditory-evoked responses - the auditory cortical P1-N1 response and the mismatch negativity (MMN) response-were elicited by synthetic speech syllables from 15 children, 11 young adults, and 10 elderly adults. Stimuli consisted of two synthesized consonant and vowel (CV) syllables along a /da/-to-/ga/ continuum that differed in the onset frequency of the third formant $\left(\mathrm{F}_{3}\right)$. The duration of both stimuli was $100 \mathrm{msec}$, with a 40 msec formant transition. The onset frequency of $F_{3}$ was 2580 and 2300 $\mathrm{Hz}$ for /da/ and /ga/, respectively. The acoustic difference between the two stimuli was easily discriminated by all subjects psychophysically.

Stimulus files were downloaded from a Klatt synthesizer to a personal computer (PC)-based stimulus delivery system that controlled the time of 
delivery and stimulus intensity and triggered the PC-based evoked potential-averaging system. Stimuli were presented at $75 \mathrm{~dB}$ sound pressure level (SPL) to the right ear of each subject through insert earphones (Etymotic ER-2) at a rate of $1.9 / \mathrm{sec}$. The use of monaural stimulus presentation was necessitated by the paradigm of the present study. First, the evoked response recording required multiple sessions of $2 \mathrm{hr}$ each. Second, the MMN response can be affected by attention (Woldorff et al., 1991; Alho et al., 1992; Woods et al., 1992). For these reasons, subjects were seated comfortably in a reclining chair and allowed to view videotapes of their choice during testing; the left ear was unoccluded, and the videotape audio levels were kept below $40 \mathrm{~dB}$ SPL (A-weighted) to not interfere with the recording and to allow the subject to hear the video soundtrack. This paradigm helped ensure that the subjects were unlikely to attend to the test stimuli because the video soundtrack was inherently more interesting while, at the same time, minimizing changes in the level of arousal throughout the test session. This paradigm also was instrumental in encouraging the subjects to sit quietly for the lengthy test sessions, because they were able to view full-length movies. In addition, the use of monaural stimulation in the evaluation of hemispheric asymmetry and topography of auditory-evoked potentials in humans is not unprecedented (Pekkonen et al., 1995), and previous research has indicated that consistent patterns of hemispheric asymmetry in the neurophysiological representation of speech signals occur regardless of whether right ear, left ear, or binaural stimulation is used (King et al., 1999). Finally, because the mode of stimulation was constant across all subjects, any differences observed in the topography of responses between subject groups could not be attributed to stimulus delivery issues.

Electrophysiological responses were obtained using a recording window that included a prestimulus baseline of $100 \mathrm{msec}$ and a poststimulus time window of $500 \mathrm{msec}$. Evoked responses were analog bandpass filtered on-line from 0.1 to $100 \mathrm{~Hz}$ (12 dB/octave roll off). Responses were recorded over the right and left temporal lobes (TR; TL) with a noncephalic (nose tip) reference. TR was located halfway between electrode sites T4 and T6, and TL was located halfway between T3 and T5 according to the international ten-twenty system (Jasper, 1958). A forehead electrode served as the ground. Eye movements were monitored with a supraorbital-to-lateral canthus bipolar electrode montage.

The MMN and P1-N1 responses were obtained using procedures that have been described previously (Kraus et al., 1996). The /ga/ and /da/ stimuli served as the standard and deviant stimuli, respectively, in an oddball paradigm. Stimuli were presented in a pseudorandom sequence with at least three standard stimuli separating presentations of deviant stimuli. The deviant probability of occurrence was $10 \%$. Twenty standard stimuli preceded the occurrence of the first deviant stimulus, and responses to standard stimuli immediately after the occurrence of a deviant stimulus were excluded from the average.

Evoked responses elicited by standard and deviant stimuli were averaged separately. For each subject, responses to $\sim 250$ deviant (/da/) stimuli were obtained along with responses to $1800-2500$ standard (/ga/) stimuli. In addition, responses to $1800-2500$ stimulus presentations of the deviant (/da/) stimulus presented alone were obtained.

\section{Speech sound discrimination stimuli and response parameters}

The speech sound discrimination procedure has been described elsewhere (Carrell et al., 1999; Kraus et al., 1999). A parameter estimation by sequential tracking (PEST) procedure was used to evaluate just noticeable differences (JNDs) for synthesized CV speech continua in 17 children, 12 young adults, and 12 older adults. Continua were created using a Klatt synthesizer and represented differences in the third formant onset frequency (/da/ to /ga/). Previous research has shown that individuals with auditory perceptual deficits and/or auditory cortex lesions exhibit deficits perceiving rapid transitions that characterize many consonants, whereas perception of slowly changing, steady-state sounds is not affected (Phillips and Farmer, 1990). An additional continuum that represented changes in the duration of the first and second formants (/ba/ to /wa/), shown to be less vulnerable to misperception (Kraus et al., 1996), also was created for use as a control to ensure that subjects understood and were capable of performing the task.

For both continua, the end points were defined by ideal examples of the syllables (Pisoni et al., 1983; Walley and Carrell, 1983). For the /da/-to-/ga/ continuum, the third formant onset frequency varied from $2580 \mathrm{~Hz}(/ \mathrm{da} /)$ to $2180 \mathrm{~Hz}(/ \mathrm{ga} /)$ in 40 steps of $10 \mathrm{~Hz}$ each. The formant transition duration was $40 \mathrm{msec}$. For the / ba/-to-/wa/ continuum, the duration of the first and second formant transition varied from $10 \mathrm{msec}$ (/ba/) to $40 \mathrm{msec}(/ \mathrm{wa} /)$ in 30 steps of $1 \mathrm{msec}$ each. Thus, a JND of 7 for the /da/-to-/ga/ task would indicate that the subject could discriminate a difference of $70 \mathrm{~Hz}$ in onset frequency of the third formant. Likewise, a JND of 7 for the /ba/-to-/wa/ task would indicate that the subject could discriminate a difference of $7 \mathrm{msec}$ in formant transition, or voice onset time. The total stimulus duration for all stimuli was $100 \mathrm{msec}$.

A four-interval, forced choice procedure was used to prevent response bias. In each trial, subjects were presented with two pairs of syllables in which one pair was the same and one pair was different. The subjects' task was to indicate via a button push in which interval pair the syllables were different. Consistent with the PEST algorithm, the acoustic difference between stimuli became smaller after correct answers and larger after incorrect answers. The order of same and different pairs within trials was randomized. The listener's JND was defined as the distance between stimuli in the "different" pair when the listener reliably reached an accuracy level of $69 \%$ correct. Three trial blocks were obtained for each stimulus condition. In our experience, individuals occasionally perform poorly on an isolated block during the test procedure because of fatigue, unfamiliarity with the task, and/or attention-related issues. Therefore, to reduce the impact of these occasional lapses in performance and to obtain a measure of the individual subject's best discrimination abilities, we computed the JND for each stimulus contrast as the mean of the two best blocks.

\section{Analysis}

P1-N1 responses. The P1 was identified as the largest positive deflection after stimulus onset in the latency region between 50 and $100 \mathrm{msec}$. N1 was identified as the negative deflection after the P1. Peak-to-peak amplitude measures of the P1-N1 complex were calculated off-line as the amplitude in microvolts from the peak of the P1 response to the negativemost point of the $\mathrm{N} 1$ response. Latency measures in milliseconds after stimulus onset also were obtained for the P1 and N1; however, because results indicated no differences in the latency of $\mathrm{P} 1$ or $\mathrm{N} 1$ between hemispheres for any subject group, these analyses are not included here. $\mathrm{P} 1-\mathrm{N} 1$ responses were evaluated using the averaged responses obtained in the standard (/ga/) and deviant-alone (/da/) conditions. The purpose of analyzing the $\mathrm{P} 1-\mathrm{N} 1$ responses to both stimuli was to determine whether the neurophysiological representation differed between stimuli and/or stimulus condition. It should be noted that the stimuli used in this study were quite similar acoustically, differing by only $280 \mathrm{~Hz}$ in $\mathrm{F}_{3}$ frequency compared with the classic exemplars of /da/ and /ga/, which differ by $400 \mathrm{~Hz}$. After preliminary analyses that revealed no significant differences in responses as a function of stimulus type (/da/ vs /ga/), results elicited by both stimuli were collapsed for all further analyses.

A two $\times$ three repeated measures ANOVA [within, side of response (right or left); between, group (children, young adult, or elderly)] was performed for peak-to-peak amplitude values to determine whether responses were asymmetric (as evidenced by a significant main effect of side of response) and whether the patterns of asymmetry differed among subject groups (as evidenced by the side $\times$ group interaction).

In addition, the degree of hemispheric asymmetry was computed by subtracting the right hemisphere peak-to-peak amplitude value from the left hemisphere peak-to-peak amplitude value and dividing by the sum of the two values: [(TL $-\mathrm{TR}) /(\mathrm{TL}+\mathrm{TR})]$. Using this equation, completely symmetrical responses would result in a value of zero, larger responses over the left hemisphere would result in positive values, and larger responses over the right hemisphere would result in negative values. Asymmetry values were subjected to a univariate ANOVA procedure to determine the effects of subject group on temporal lobe asymmetry.

$M M N$ responses. The MMN is elicited by a deviant stimulus only when it signals an acoustic change. Therefore, difference waves were computed for each subject by subtracting the response to the deviant stimuli presented alone from the response to the deviant stimuli presented within the oddball paradigm (Alho et al., 1989; Kraus et al., 1995). MMN responses were identified visually in the difference wave as a relative negativity after the $\mathrm{N} 1$ and occurring in the latency range of 100-500 msec. Onset, peak, and offset latencies were measured. MMN duration was computed by subtracting the onset latency from the offset latency. Amplitude of the onset-to-peak latency was obtained, and the response area was computed by integrating the overall area between the onset and offset latencies.

As with the $\mathrm{P} 1-\mathrm{N} 1$ responses, a two $\times$ three repeated measures ANOVA was conducted for MMN amplitude and area values to determine whether the response magnitude was asymmetric and whether the degree of asymmetry differed among subject groups.

Fine-grained speech sound discrimination. Univariate ANOVAs were 


\section{P1-N1 Hemispheric Asymmetry}

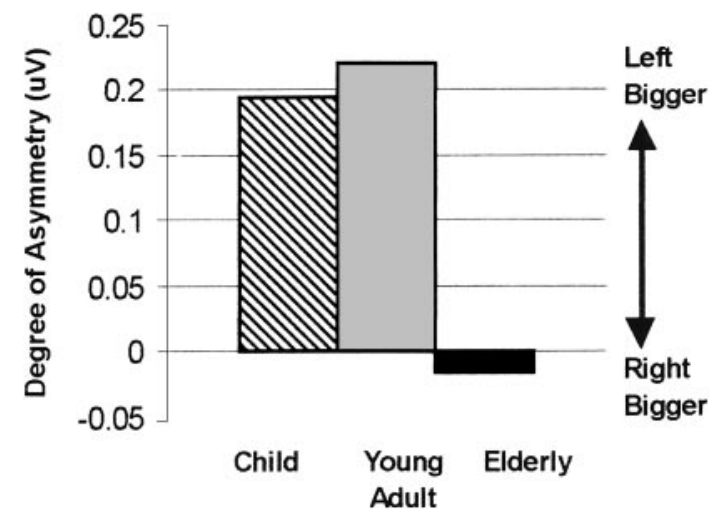

Figure 1. Effect of age on the degree of temporal lobe asymmetry in the $\mathrm{P} 1-\mathrm{N} 1$ response as calculated by the equation [(TL $-\mathrm{TR}) /(\mathrm{TL}+\mathrm{TR})]$. Results indicate that children and young adults demonstrated a significantly greater degree of temporal lobe asymmetry favoring the left hemisphere than did older adults. For older adults, responses were essentially symmetrical.

conducted for subjects' mean JND scores for each stimulus contrast to determine whether the ability to discriminate the stimuli differed among subject groups.

\section{RESULTS}

\section{P1-N1 response asymmetry}

ANOVA revealed a significant main effect of subject group on the degree of temporal lobe asymmetry $(F=5.517 ; p<0.01)$. Post hoc Bonferroni comparisons revealed that children and young adults exhibited a significantly greater degree of temporal lobe asymmetry than did the elderly subjects who exhibited essentially symmetrical responses $(p<0.01)$. These results are illustrated in Figure 1.

Figure 2 shows the grand averages of P1-N1 responses obtained over the right and left temporal lobes for the three subject groups. This figure clearly shows that responses recorded over the left hemisphere were substantially larger than those recorded over the right hemisphere for the children and young adult subjects. Although left hemisphere responses appear somewhat larger than right hemisphere responses in the grand averages for the elderly group, as well, this difference did not approach statistical significance. This underscores the necessity of using individual data points and statistical analysis in any event-related potential study, because the pictorial representation of responses via grand averages inherently are limited in that a large-magnitude response of even a single individual may be over-represented because of the relatively greater weighting afforded larger responses in the grand average.

Hemispheric asymmetry data obtained from individual subjects in each age group using the equation $[(\mathrm{TL}-\mathrm{TR}) /(\mathrm{TL}+$ TR)] are displayed in Figure 3. As can be seen from this figure, the majority of children and all of the young adult subjects exhibited asymmetry of response amplitude favoring the left side. Overall, the elderly subjects exhibited symmetrical responses.

\section{P1-N1 peak-to-peak amplitude}

Results of repeated measures ANOVA revealed significant main effects of side of response $(F=16.274 ; p<0.01)$ and group $(F=$ 9.646; $p<0.01)$ on $\mathrm{P} 1-\mathrm{N} 1$ peak-to-peak amplitude, as well as a

\section{Temporal Lobe Responses Grand Averages}

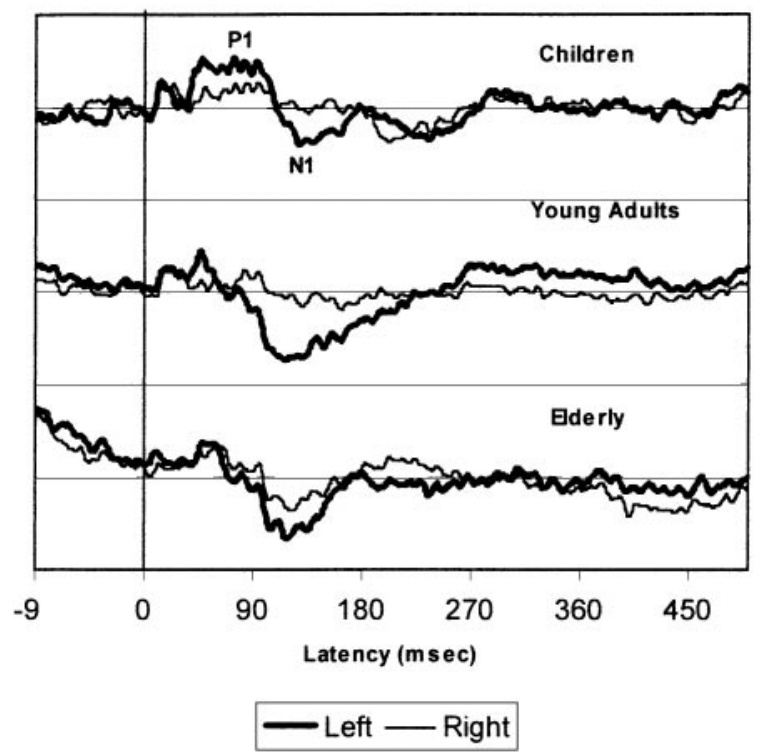

Figure 2. Grand averaged P1-N1 responses from the right and left temporal lobe electrode sites for children $(n=15)$, young adults $(n=11)$, and older adult subjects $(n=10)$. Responses were elicited by $1800-2500$ repetitions of synthetic /da/ and /ga/ stimuli presented to the right ear at an intensity of $75 \mathrm{~dB}$ SPL and a rate of $1.9 / \mathrm{sec}$. Grand averages indicate larger responses over the left temporal lobe than over the right for children and young adults and essentially symmetrical responses for older adults.

significant side $\times$ group interaction $(F=5.557 ; p<0.01)$. When collapsed across subject groups, responses were larger over the left temporal lobe than over the right. Post hoc Bonferroni comparisons revealed that the overall response amplitude was significantly larger in the children than in the young adult or elderly subjects $(p<0.01)$. There was no difference in response amplitude between the young adult and elderly subject groups. Finally, for children and young adults, response amplitudes over the left temporal lobe were significantly larger than that over the right temporal lobe (paired $t, p<0.01$ ). In elderly subjects, right and left temporal lobe responses were symmetrical. These results are illustrated in Figure 4.

\section{MMN response symmetry}

There were no significant differences in the amplitude, duration, or area of the MMN responses obtained over right and left temporal lobes for any subject group, indicating that the MMN response is symmetrical over the temporal lobes and does not vary with age. Results of this analysis are illustrated in Figure 5.

\section{Fine-grained speech sound discrimination}

Results of univariate ANOVA procedures revealed a significant effect of subject group on the ability to discriminate the /da-ga/ stimulus contrast $(F=4.071 ; p<0.05)$. Post hoc analysis revealed that the elderly subjects exhibited significantly poorer ability to discriminate the /da-ga/ stimulus contrast compared with both the children and the young adult subjects $(p<0.05)$. That this finding was not caused by an inability to understand or perform the task is evidenced by the finding of no effect of subject group on the ability to discriminate the /ba-wa/ stimulus contrast. These results are illustrated in Figure 6. 
Children

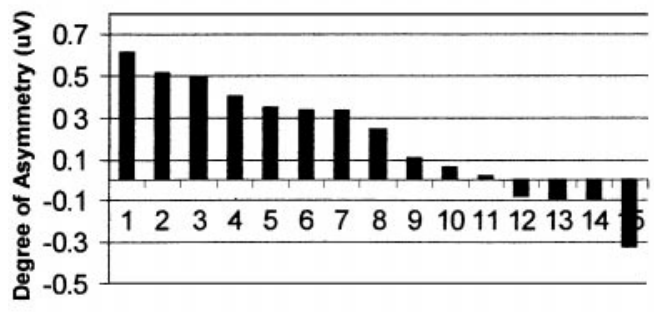

Young Adults

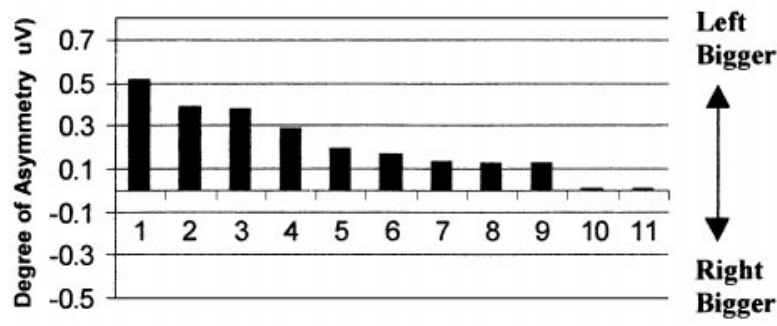

Elderly Adults

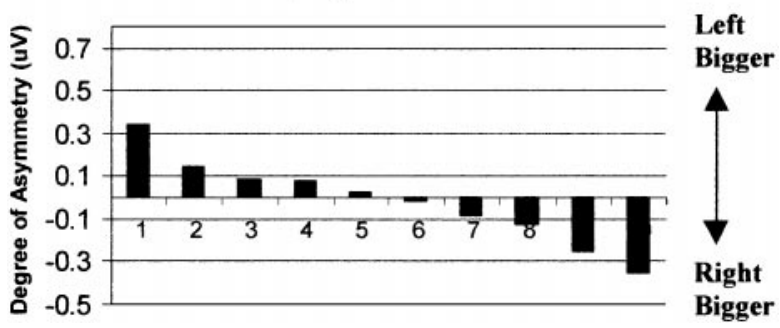

Figure 3. Individual subject data from children $(n=15)$, young adults $(n=11)$, and older adults $(n=10)$ for the degree of hemispheric asymmetry in the P1-N1 response. The degree of asymmetry was calculated using the equation [(TL $-\mathrm{TR}) /(\mathrm{TL}+\mathrm{TR})]$. Positive values indicate larger responses over the left temporal lobe; negative values indicate larger responses over the right temporal lobe. The majority of children and all of the young adult subjects exhibited larger responses over the left temporal lobe. Overall, older adults exhibited symmetrical responses.

\section{DISCUSSION}

Results of this study show that aging affects the degree of left/ right hemispheric asymmetry in the basic neural representation of speech sounds in normal hearing individuals. It has been demonstrated that left-sided specialization occurs for sounds that have complex speech-like acoustic properties (Belin et al., 1998). The dominance of the left hemisphere for processing acoustic stimuli that have rapid spectrotemporal changes, such as consonants, with a high degree of temporal precision has been demonstrated by a number of investigators (Efron, 1963; Lackner and Teuber, 1973; Schwartz and Tallal, 1980; Phillips and Farmer, 1990). Furthermore, neural and perceptual processing of the rapid acoustic transitions that characterize many consonants appears to be critical for normal speech perception and is particularly vulnerable to disruption (Godfrey et al., 1981; Elliot et al., 1989; Phillips and Farmer, 1990; Tallal, 1994; Merzenich et al., 1996). Therefore, it may be hypothesized that changes in hemispheric asymmetry as seen in the present study may have an adverse effect on the ability to process complex, rapidly changing acoustic stimuli, ultimately resulting in speech perceptual difficulties. In addition, because no
P1-N1 Amplitude

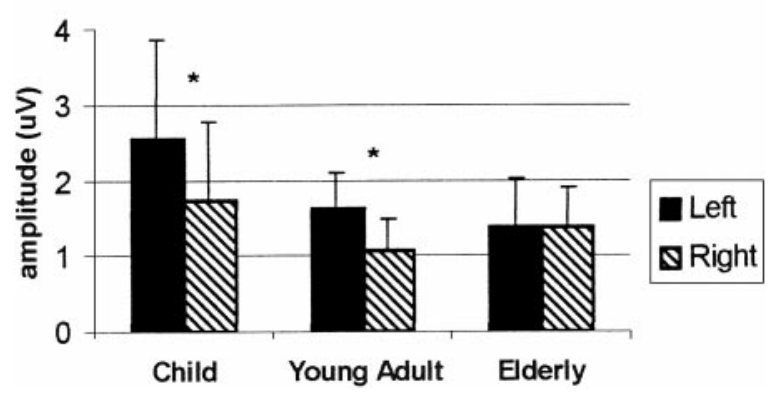

Figure 4. Effect of age group on the P1-N1 peak-to-peak amplitude obtained over right and left temporal electrode sites. Error bars represent one SD. Responses were significantly larger over the left temporal lobe than over the right for children and young adults but were symmetrical for older adults. Response amplitudes were significantly larger overall for children compared with young adult and older adult subjects.

hemispheric latency differences in neurophysiological responses were found in any group, it can be assumed that age-related changes in hemispheric asymmetry are manifested primarily in the number of neurons recruited, or the degree of hemispheric activation, rather than in the relative timing of neural transmission.

There is ample evidence to suggest that auditory temporal processing is poorer in aging listeners compared with younger adults (Humes and Christopherson, 1991; Fitzgibbons and Gordon-Salant, 1994; Divenyi and Haupt, 1997; Gordon-Salant and Fitzgibbons, 1999). Results of the present study also demonstrate that the discrimination of speech sounds involving rapid spectrotemporal acoustic change (i.e., /da-ga/) is poorer in elderly listeners, whereas the ability to discriminate speech sounds differing only in formant duration (i.e., /ba-wa/) is unaffected. These results suggest that age-related alterations in hemispheric asymmetry in the neural representation of elemental speech sounds may be one possible contributing factor to the temporalprocessing difficulties exhibited by aging adults. However, although the behavioral and physiological responses discussed in this paper are related in that they reflect processing of acoustic events, it must be remembered that they are inherently different responses. Psychophysical tasks require a conscious, behavioral response and may be affected by many different factors, including attention, ability to perform the task, stimulus and response parameters, and other factors that affect the individual's conscious perceptual abilities. In contrast, the neurophysiological response is a preattentive neural representation of acoustic events, originating primarily within the auditory pathway and independent of attention or voluntary response. In addition, alteration in the hemispheric asymmetry of speech sound representation with aging likely is just one of many factors that contribute to speech perceptual difficulties in the elderly. As such, one would not expect a direct one-to-one correlation within individuals between perceptual ability and degree of hemispheric asymmetry as reflected in the P1-N1 neurophysiological response. Nevertheless, behavioral and physiological measures reflect intersecting processes, and the findings of the present study demonstrate how these measures provide insight into specific neurophysiological processes that, at least in part, underlie psychophysical performance.

Belin et al. (1998) demonstrated that the hemispheric asymme- 


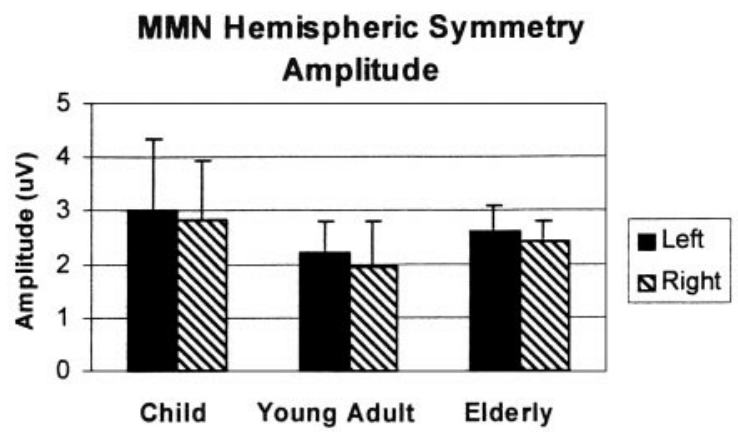

Duration
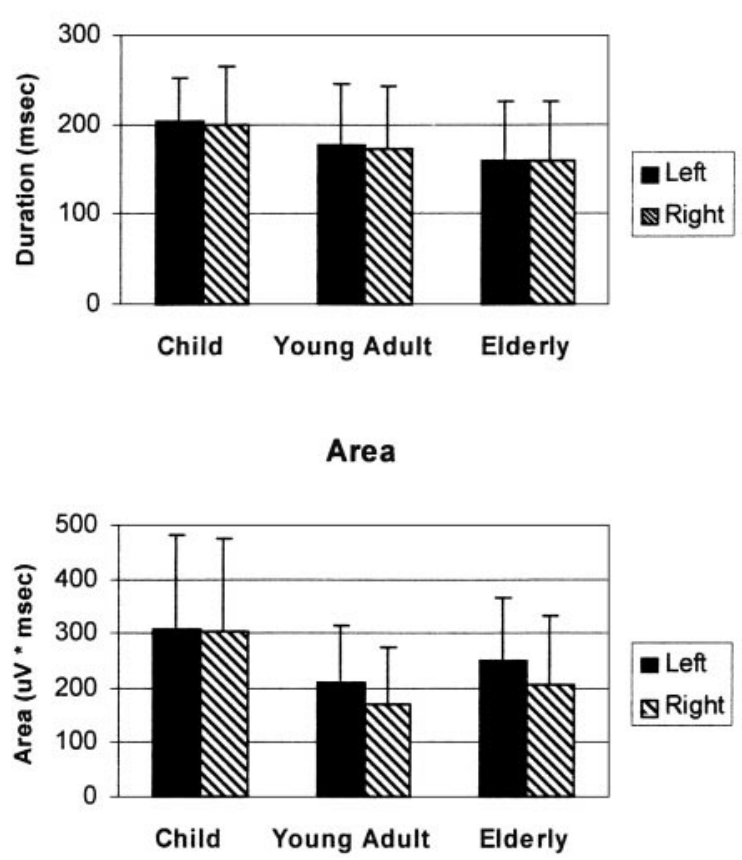

Figure 5. MMN response amplitude, duration, and area obtained over the right and left temporal lobes from children, young adults, and older adults. Error bars represent one SD. Results indicate symmetrical responses over the temporal lobes for all MMN parameters.

try of cortical activation in response to complex acoustic stimuli results from a relative decrease in right hemisphere activation during rapid acoustic change rather than from greater left hemisphere activation. Examination of the grand averaged responses in Figure 2 also suggests that the changes in hemispheric asymmetry with age in the present study may be the result of relatively greater right hemisphere activation in the elderly subjects compared with the younger two age groups. Alternatively, because the $\mathrm{P} 1-\mathrm{N} 1$ responses obtained in this study demonstrate a decline in amplitude as a function of aging, the relatively greater right hemisphere activation in the elderly subjects may be attributable to a cessation of this decline in the right hemisphere, perhaps as a result of decreased subcortical and cortical inhibition in the older population (Amenedo and Diaz, 1998). Although this topic warrants further investigation, it is possible that the apparently greater right hemisphere response to speech stimuli in aging individuals may contribute to hemispheric competition in the processing of speech, resulting in temporal blurring and concomitant speech perceptual difficulties (Hammond, 1982).
JNDs: /ba-wal

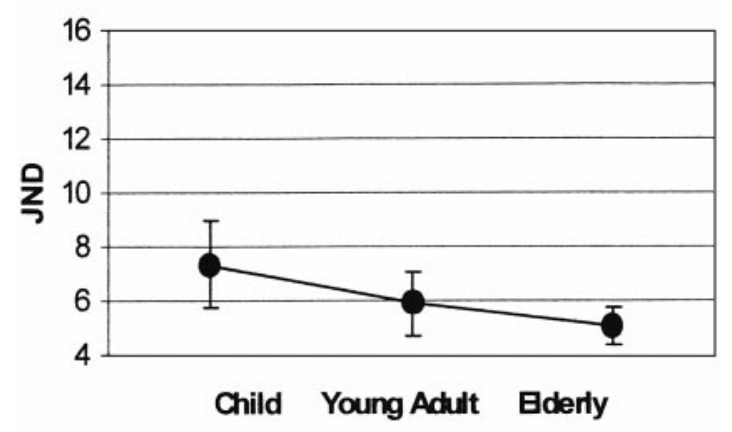

JNDs: /da-gal

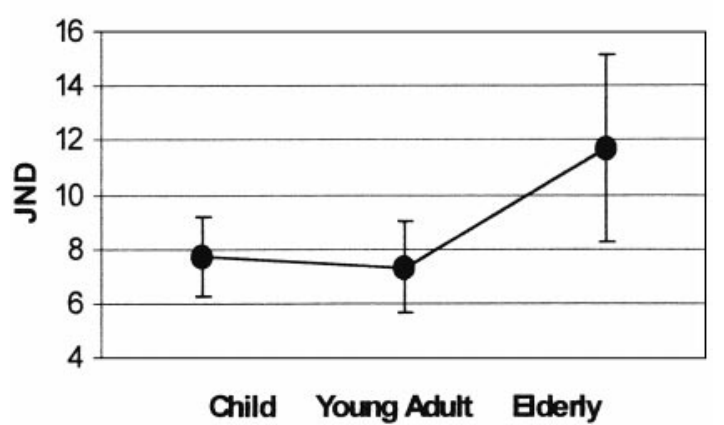

Figure 6. JNDs obtained for /da/-to-/ga/ and /ba/-to-/wa/ stimulus continua obtained from children $(n=17)$, young adults $(n=12)$, and older adults $(n=12)$. Bottom, For the /da/-to-/ga/ continuum, each JND point represents a difference of $10 \mathrm{~Hz}$ in frequency of the third formant. Top, For the /ba/-to-/wa/ continuum, each JND point represents a difference of $1 \mathrm{msec}$ in the duration of the first and second formant transition. Results indicate that elderly adults exhibit a significantly poorer ability to discriminate the /da-ga/ contrast compared with children and young adults as evidenced by the larger JNDs for the older subject group. There was no effect of subject group on the ability to discriminate the /ba-wa/ contrast.

An alternative explanation for the present findings is the possibility of an age-related change in generator sites. Because the present study involved only electrode locations placed over the right and left temporal lobes, subtle age-related shifts in generator sites for the P1-N1 would not be identified. Furthermore, although it is possible that generator sites for scalp-recorded evoked potentials may shift as a function of maturation in children, primarily because of an increase in head size, a shift in generator sites is not a likely explanation for the age-related changes in the $\mathrm{P} 1-\mathrm{N} 1$ response asymmetry in the present study for two reasons. First, significant differences were found in the topography of responses between the two adult groups in which head growth is not a factor. Second, Pekkonen et al. (1995) have demonstrated that the generator sites for the P50m and N100m (the auditory-evoked magnetometric analogs to the P1 and N1 responses examined in the present study) do not change as a function of aging from young adulthood through the age of 86 years. Thus, age-related shifts in generator sites cannot account for topographical differences in the responses of the older subjects compared with the two younger groups.

Although no statistically significant difference was found in the 
hemispheric asymmetry of the neurophysiological responses of children compared with young adults, individual subject data presented in Figure 3 indicate that several of the children in this study $(26 \%)$ exhibited greater activation over the right versus the left hemisphere. This pattern is entirely consistent with anatomical and neurophysiological data indicating a maturation of the auditory, language, and interhemispheric pathways from childhood to early adulthood (Salamy, 1978; Paus et al., 1999), followed by a regression to a more childlike degree of myelination and anatomical structure in later years (Allen et al., 1991; Hanyu et al., 1997).

Finally, the findings of King et al. (1999) indicate that left/right asymmetry in the neural representation of speech stimuli is apparent even at the thalamic level of the central auditory pathway of nonhuman mammals. Thus, such asymmetry appears to be a basic, prelinguistic element of normal speech perception, and alterations in the pattern and/or degree of asymmetry with aging may hold critical implications for the processing of elemental speech features in the aging adult.

In contrast, acoustic change appears to be bilaterally represented, as evidenced by hemispheric symmetry in the MMN response for all subject groups. Previous research has indicated that, whereas the P1-N1 response demonstrates morphological changes from infancy through the second decade of life (Courchesne, 1990; Ponton et al., 1996; Cunningham et al., 1997), the MMN remains stable and demonstrates no developmental changes throughout the school-age years (Kraus et al., 1999). This finding, combined with evidence demonstrating that the $\mathrm{MMN}$ is generated by sources in the nonprimary thalamus and auditory cortex (Scherg et al., 1989; Kraus et al., 1994) that are different from those that generate the cortical auditory-evoked N1 response (Naatanen and Picton, 1987; Sams et al., 1991), supports the theory that the MMN and P1-N1 responses represent different neurophysiological processes. Specifically, the P1-N1 response appears to reflect the basic neural encoding of repetitive, identical acoustic stimuli primarily in the primary auditory pathways (Eberling et al., 1982; Pantev et al., 1998), whereas the MMN likely reflects a preperceptual echoic or trace memory process related to the representation of acoustic change (Naatanen et al., 1989) that is bilaterally represented and mediated by nonprimary auditory pathways. Results of the present study indicate that age affects the basic neural representation of speech sounds but has no effect on the neural representation of acoustic change.

In conclusion, these results demonstrate that the pattern of left-sided dominance in the neural representation of speech sounds seen in children and young adults is not evident in older adults, despite normal hearing sensitivity. This absence of hemispheric asymmetry is accompanied by a poorer ability to discriminate speech sounds involving rapid spectrotemporal changes in older adult listeners. These findings provide evidence of a biological, age-related change in the basic sensory representation of elemental speech signals and suggest one possible underlying mechanism for the speech perceptual difficulties experienced by aging adults. Results of this study also provide a normal metric for comparison with other populations exhibiting communicative difficulties, including individuals with auditory-processing deficits, so that functional implications of atypical patterns of hemispheric asymmetry may be delineated. Finally, results of this study support previous evidence suggesting that auditory processes reflected by the $\mathrm{P} 1-\mathrm{N} 1$ response and the $\mathrm{MMN}$ are mediated by different neural generators and reflect separate neurophysiological and functional mechanisms.

\section{REFERENCES}

Alho K, Sams M, Paavilainen P, Reinikainen K, Naatanen R (1989) Event-related brain potentials reflecting processing of relevant and irrelevant stimuli during selective listening. Psychophysiology 26:514-528.

Alho L, Woods D, Algazi A, Naatanen R (1992) Intermodal selective attention. II. Effects of attentional lead on processing of auditory and visual stimuli in central space. Electroencephalogr Clin Neurophysiol 82:356-368.

Allen LS, Richey MF, Chai YM, Gorski RA (1991) Sex differences in the corpus callosum of the living human being. J Neurosci 11:933-942.

Amenedo E, Diaz F (1998) Effects of aging on middle-latency auditory evoked potentials: a cross-sectional study. Biol Psychiatry 43:210-219.

Auerbach SH, Allard T, Naeser M, Alexander MP (1982) Pure word deafness. Analysis of a case with bilateral lesions and a defect at the prephonemic level. Brain 105:271-300.

Belin P, Zilbovicius M, Crozier S, Thivard L, Fontaine A, Masure M-C, Samson Y (1998) Lateralization of speech and auditory temporal processing. J Cognit Neurosci 10:536-540.

Carrell T, Bradlow A, Nicol T, Koch D, Kraus N (1999) Interactive software for evaluating auditory discrimination. Ear Hear 20:175-176.

Courchesne E (1990) Chronology of postnatal human brain development: event related potential, positron emission tomography, myelinogenesis and synaptogenesis studies. In: Event related potentials (Rohrbaugh J, Parasuraman R, Johnson R, eds), pp 195-206. New York: Oxford.

Cunningham J, Nicol T, Bradlow A, McGee T, Kraus N (1997) Speechevoked $\mathrm{P} 1 / \mathrm{N} 1$ response in normal and learning disabled children. ARO Abstract 101.

Dawson G, Finley C, Phillips S, Lewy A (1989) A comparison of hemispheric asymmetries in speech-related brain potentials of autistic and dysphasic children. Brain Lang 14:26-41.

Divenyi PL, Haupt KM (1997) Audiological correlates of speech understanding deficits in elderly listeners with mild-to-moderate hearing loss. I. Age and lateral asymmetry effects. Ear Hear 18:42-61.

Eberling C, Bak C, Kofoed B, Lebech J, Saermark K (1982) Auditory magnetic fields. Source location and tonotopical organization of the right hemisphere of the human brain. Scand Audiol 9:203-207.

Efron R (1963) Temporal perception, aphasia and $\mathrm{d} \theta \mathrm{j} \alpha$ vu. Brain 86:403-424.

Elliot LL, Hammer MA, Scholl ME (1989) Fine-grained auditory discrimination in normal children and children with language-learning problems. J Speech Hear Res 31:112-119.

Elmo T (1987) Hemispheric asymmetry of auditory evoked potentials to comparisons within and across phonetic categories. Scand J Psychol 28:251-266.

Fitch RH, Brown CP, O’Connor K, Tallal P (1993) Functional lateralization for auditory temporal processing in male and female rats. Behav Neurosci 107:844-850.

Fitzgibbons PJ, Gordon-Salant S (1994) Age effects on measures of auditory duration discrimination. J Speech Hear Res 37:662-670.

Godfrey JJ, Syrdal-Lasky AK, Millay KK, Knox CM (1981) Performance of dyslexic children on speech perception tests. J Exp Child Psychol 32:401-424.

Gordon-Salant S, Fitzgibbons PJ (1999) Profile of auditory temporal processing in older listeners. J Speech Hear Res 42:300-311.

Hammond GR (1982) Hemispheric differences in temporal resolution. Brain Cogn 1:95-118.

Hanyu H, Asano T, Ogawa K, Takasaki M, Shindo H, Kakizaki D, Abe D (1997) Age-related changes of diffusional anisotropy in the cerebral white matter in normal subjects. No To Shinkei 49:331-336.

Humes L, Christopherson L (1991) Speech identification difficulties of hearing-impaired elderly persons: the contributions of auditory processing deficits. J Speech Hear Res 34:686-693.

Jasper HH (1958) The ten-twenty system of the International Federation. Electroencephalogr Clin Neurophysiol 10:371-375.

Jerger J, Jordan C (1992) Age increases asymmetry on a cued-listening task. Ear Hear 13:272-277.

Jerger J, Chmiel R, Allen J, Wilson A (1994) Effects of age and gender on dichotic sentence identification. Ear Hear 15:274-286.

King C, Nicol T, McGee T, Kraus N (1999) Thalamic asymmetry is 
related to acoustic signal complexity in guinea pigs. Neurosci Lett 267:89-92.

Kraus N, McGee T, Littman T, Nicol T, King C (1994) Nonprimary auditory thalamic representation of acoustic change. J Neurophysiol 72:1270-1277.

Kraus N, McGee T, Carrell T, Sharma A, Nicol T (1995) Mismatch negativity to speech stimuli in school age children. Electroencephalogr Clin Neurophysiol 44:211-217.

Kraus N, McGee TJ, Carrell TD, Zecker ZD, Nicol TG, Koch DB (1996) Auditory neurophysiologic responses and discrimination deficits in children with learning problems. Science 273:971-973.

Kraus N, Koch DB, McGee TJ, Nicol TG, Cunningham J (1999) Speechsound discrimination in school-age children: psychophysical and neurophysiologic measures. J Speech Hear Res 5:1042-1060.

Lackner JR, Teuber H-L (1973) Alterations in auditory fusion thresholds after cerebral injury in man. Neuropsychologia 11:409-415.

Marvel JB, Jerger JF, Lew HL (1992) Asymmetries in topographic brain maps of auditory evoked potentials in the elderly. J Am Acad Audiol 3:361-368.

Merzenich M, Jenkins W, Johnston P, Schreiner C, Miller S, Tallal P (1996) Temporal processing deficits of language-learning impaired children ameliorated by training. Science 271:77-81.

Naatanen R, Picton TW (1987) The N1 wave of the human electric and magnetic response to sound: a review and an analysis of the component structure. Psychophysiology 24:375-425.

Naatanen R, Paavilainen P, Rein I, Kainen K (1989) Do event-related potentials to infrequent decrements in duration of auditory stimuli demonstrate a memory trace in man? Neurosci Lett 107:347-352.

Obrzut JE, Hynd GW, Zellner RD (1983) Attentional deficit in learning-disabled children: evidence from visual half-field asymmetries. Brain Cogn 2:89-101.

Pantev C, Ross B, Berg P, Elbert T, Rockstroh B (1998) Study of the human auditory cortices using a whole-head magnetometer: left vs. right hemisphere and ipsilateral vs. contralateral stimulation. Audiol Neurootol 3:183-190.

Paus T, Zijdenbos A, Worsley K, Collins DL, Blumenthal J, Gledd JN, Rapoport JL, Evans AC (1999) Structural maturation of neural pathways in children and adolescents: in vivo study. Science 283:1908-1911.

Pekkonen E, Huotilainen M, Virtanen J, Sinkkonen J, Rinne T, Ilmoni- emi RJ, Naatanen R (1995) Age-related functional differences between auditory cortices: a whole-head MEG study. NeuroReport 6:1803-1806.

Phillips DP, Farmer ME (1990) Acquired word deafness and the temporal grain of sound representation in the primary auditory cortex. Behav Brain Res 40:85-94.

Pisoni D, Carrell T, Gans J (1983) Perception of the duration of rapid spectrum changes in speech and nonspeech signals. Percept Psychophys 34:314-322.

Ponton C, Don M, Eggermont J, Waring M, Kwong B, Masuda A (1996) Auditory system plasticity in children after long periods of complete deafness. NeuroReport 8:61-65.

Salamy A (1978) Commissural transmission: maturational changes in humans. Science 200:1409-1410.

Sams M, Kaukoranta E, Hamalainen M, Naatanen R (1991) Cortical activity elicited by changes in auditory stimuli: different sources for the magnetic N100m and mismatch responses. Psychophysiology 28:21-29.

Scherg M, Vajsar J, Picton TW (1989) A source analysis of the late human auditory evoked potentials. J Cognit Neurosci 1:336-355.

Schwartz J, Tallal P (1980) Rate of acoustic change may underlie hemispheric specialization for speech perception. Science 207:1380-1381.

Sharma A, Kraus N, Carrell T, Thompson C (1994) Physiologic bases of pitch and place of articulation perception: a case study. J Acoust Soc Am 95:3011-3019.

Tallal P (1994) In the perception of speech time is of the essence. In: Temporal coding in the brain (Buzsaki G, Llinas R, Singer W, Berthoz A, Christen Y, eds). Berlin: Springer.

Walley AD, Carrell TD (1983) Onset spectra and formant transition in the adult's and child's perception of place of articulation in stop consonants. J Acoust Soc Am 73:1011-1022.

Woldorff M, Hackley S, Hillyard S (1991) The effects of channelselective attention on the mismatch negativity wave elicited by deviant tones. Psychophysiology 28:30-42.

Woods D, Alho K, Algazi A (1992) Intermodal selective attention. I. Effects on event-related potentials to lateralized auditory and visual stimuli. Electroencephalogr Clin Neurophysiol 82:341-355.

Zatorre RJ, Evans AC, Meyer E, Gjedde A (1992) Lateralization of phonetic and pitch discrimination in speech processing. Science 256: 846-849. 